\title{
Early detection of anthracycline-induced cardiotoxicity using two-dimensional speckle tracking echocardiography
}

\author{
Yu Kang ${ }^{1,2 *}$, Leilei Cheng ${ }^{2 *}$, Lin $\mathrm{Li}^{2}$, Haiyan $\mathrm{Chen}^{2}$, Minmin $\mathrm{Sun}^{2}$, \\ Zheng Wei ${ }^{3}$, Cuizhen $\mathrm{Pan}^{2}$, Xianhong Shu ${ }^{2}$ \\ ${ }^{1}$ Department of Cardiology, Renji Hospital of Jiaotong University, Shanghai, China \\ ${ }^{2}$ Department of Echocardiography, Zhongshan Hospital of Fudan University, \\ Shanghai Institute of Cardiovascular Disease, Shanghai, China \\ ${ }^{3}$ Department of Hematology, Zhongshan Hospital of Fudan University, Shanghai, China
}

\begin{abstract}
Background: To examine the subclinical myocardial injury shortly after epirubicin exposure in asymptomatic patients with large B-cell non-Hodgkin lymphoma using 2-dimensional (2D) speckle tracking echocardiography.

Methods: Sixty-seven patients aged $52.58 \pm 13.86$ years with large B-cell non-Hodgkin lymphoma treated with epirubicin were studied. Echocardiography was performed at baseline and 1 day after completion of the therapy. Global and regional longitudinal (LS), circumferential $(C S)$ and radial strain $(R S)$, standard deviation of time to peak $L S\left(T_{L S-S D}\right), C S\left(T_{C S-S D}\right), R S$ $\left(T_{R S-S D}\right)$ were calculated using $2 D$ speckle tracking echocardiography.

Results: Despite normal left ventricular ejection fraction, global LS $(-18.30 \pm 1.87 \%$ vs. $-16.18 \pm 1.92 \% ; p<0.01), C S(-20.37 \pm 2.89 \%$ vs. $-18.25 \pm 2.40 \%, p<0.01)$ and $R S$ $(39.95 \pm 5.79 \%$ vs. $36.15 \pm 5.79 \%, p<0.01)$, were remarkably reduced compared to baseline values. However, $T_{L S-S D}, T_{C S-S D}$ and $T_{R S-S D}$ showed no significant difference after epirubicin exposure. Hypertension was an independent predictor of reduction of global LS, CS and RS.

Conclusions: Subtle abnormalities in myocardial systolic function were present in asymptomatic patients shortly after anthracycline exposure, which could be detected by $2 D$ speckle tracking echocardiography. (Cardiol J 2013; 20, 6: 592-599)
\end{abstract}

Key words: two-dimensional speckle tracking echocardiography, anthracycline-induced cardiotoxicity, left ventricular systolic function

\section{Introduction}

Anthracyclines, such as doxorubicin and epirubicin, remain the preferred cytotoxic agents for a wide variety of malignancies. However, anthracyclines are also frequently associated with adverse effects, of which the most important is car- diotoxicity, predominantly manifested as a dilated cardiomyopathy $[1,2]$. With the increasing number of long-term survivors resulting from successful antitumor treatment, identifying reliable non-invasive methods for the detection of myocardial dysfunction is therefore crucial. In clinical practice, serial monitoring of left ventricular ejection fra-

Address for correspondence: Xianhong Shu, PhD, Department of Echocardiography, Zhongshan Hospital of Fudan University, Shanghai Institute of Cardiovascular Disease, No. 180 Fenglin Rd Shanghai China, tel: +862164041990,

e-mail:shu.xianhong@zs-hospital.sh.cn

*These authors contributed equally to this work. 
ction (LVEF) using noninvasive cardiac imaging is the most important clinical diagnostic tool in early recognition of cardiac dysfunction [3, 4]. However, they are somewhat insensitive in detecting early signs of cardiac stress, myocardial injury, and changes in myocardial compliance. A reduction in LVEF usually occurs in the later stage of chronic pathology, followed by a course of progressive irreversible myocardial damage with poor outcomes.

Currently, two-dimensional (2D) speckle tracking echocardiography, based on tracking local image details from frame to frame throughout the cardiac cycle, is most frequently used for the assessment of LV mechanics [5-8]. It has allowed more accurate measurements of regional myocardial systolic performance.

In this study we sought to assess the subclinical myocardial dysfunction in patients before and after receiving epirubicin treatment with speckle tracking echocardiography.

\section{Methods}

\section{Study population}

A total of 73 patients with newly diagnosed, histopathologically confirmed large B-cell non-Hodgkin lymphoma in our hospital were enrolled in this study between March 2011 and May 2012. Exclusion criteria were: uncontrolled hypertension, significant valvular disease, conge-nital disease, a widened QRS complex on surface ECG, arrhythmia, a previous history of heart failure and/or coronary artery disease. All patients were treated with R-CHOP (cyclophosphamide $750 \mathrm{mg} / \mathrm{m}^{2}$, vincristine $1.4 \mathrm{mg} / \mathrm{m}^{2}$ up to a maximum dose of $2 \mathrm{mg}$, epirubicin $50-70 \mathrm{mg} / \mathrm{m}^{2}$ on day 1 , prednisone $100 \mathrm{mg}$ on days $1-5$ ), and rituximab $375 \mathrm{mg} / \mathrm{m}^{2}$. All therapy was given intravenously, except for prednisone, which was administered orally. Cycles were administered every 21 days for a maximum of 8 cycles. Rituximab was administered before the chemotherapy. Patients were restaged after cycle 3 , and those with disease progression were withdrawn from the treatment of R-CHOP. No patient received other cardiotoxic agents or radiation therapy during this study. Echocardiography was performed at baseline and 1 day after completion of the therapy. All subjects gave informed consent, and the Zhongshan Hospital Research Ethics Committee approved the protocol.

\section{Echocardiographic imaging}

Images were obtained in the left lateral decubitus position with a commercially available system
(iE33, Philips Medical Systems, N.A., Bothell, WA, USA) equipped with S5-1 transducer. Standard 2D images were acquired according to recommendations of the American Society of Echocardiography [9]. Depth was minimized to optimize the frame rate. At least 3 beats were digitally stored for offline analysis. LVEF was calculated using the modified Simpson's biplane method. LV end-diastolic dimensions, LV end-systolic dimensions, transmitral peak early (E) and peak late (A) diastolic filling velocities were also measured.

\section{Speckle tracking echocardiography}

Gray scale images for offline speckle tracking analysis were acquired at frame rate of 53 to $72 \mathrm{MHz}$. QLAB version 8.1 (Philips Medical System, N.A., Bothell, WA, USA) was used for strain analyses. The automatic tracking analysis was performed in the apical 4-chamber, 2-chamber, apical LV longitudinal view for longitudinal strain (LS) and in the parasternal short-axis view at basal and mid-papillary level for circumferential (CS) and radial strain (RS) according to the vendor's instructions. The endocardial border was manually traced at end-diastole. Tracking was automatically performed, and if tracking was suboptimal, the endocardial border was retraced. If satisfactory tracking was not accomplished within 5 min, the non-tracking segments were excluded from analysis (Fig. 1).

Peak LS measurements were obtained from the basal, mid-segments of the anterior, inferior, anteroseptal, anterolateral, inferoseptal, inferolateral walls, apical segments of anterior, inferior, septal, lateral walls and apex, totally 17 segments. Peak $\mathrm{CS}$ and RS measurements were obtained from the basal, mid-segments of the anterior, inferior, anteroseptal, anterolateral, inferoseptal, inferolateral walls, totally 12 segments. Regional strain values were averaged to determine global LS/CS/RS.

Standard deviation of the time to negative peak value of $\mathrm{LS}$ for 17 segments $\left(\mathrm{T}_{\mathrm{LS} \text {-SD }}\right)$, of the time to negative peak value of CS for 12 segments $\left(\mathrm{T}_{\mathrm{CS}-\mathrm{SD}}\right)$ and of the time to positive peak value of RS for 12 segments $\left(\mathrm{T}_{\mathrm{RS} \text {-SD }}\right.$ ) were calculated to evaluate $\mathrm{LV}$ dyssynchrony. $\mathrm{T}_{\text {LS-SD }}, \mathrm{T}_{\text {CS-SD }}$ and $\mathrm{T}_{\text {RS-SD }}$ were expressed as a percentage of the duration of the cardiac cycle.

\section{Reproducibility}

Intra- and inter-observer reproducibility was assessed by calculating the difference between the values of 10 randomly selected patients measured by 1 observer twice and by a second observer. 


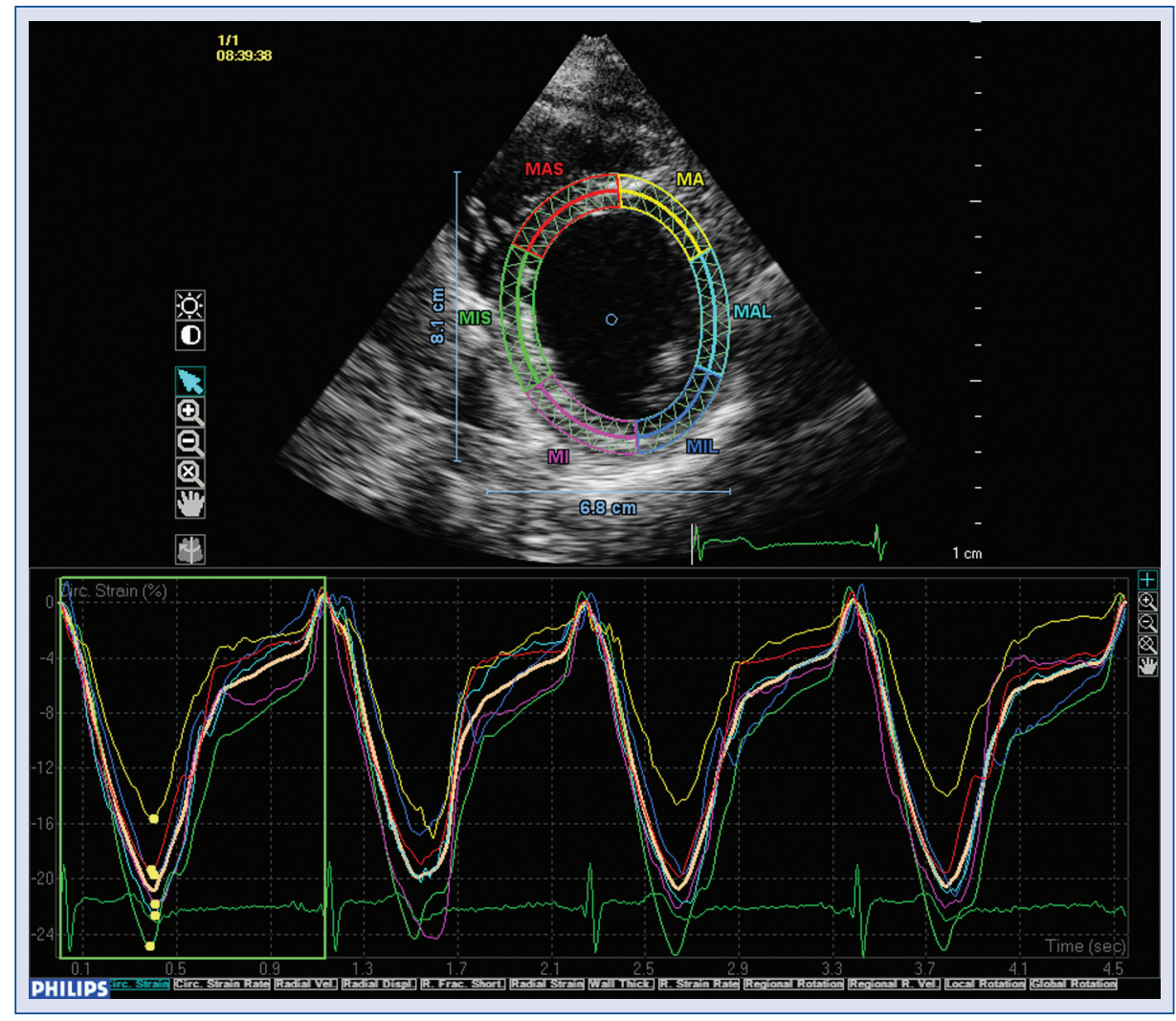

Figure 1. Mid-papillary level of parasternal short-axis view, speckle tracking-derived circumferential strain. Custom software calculates circumferential strain by tracking multiple acoustic speckles in the myocardium. It is divided into 6 segments: anterior, anteroseptal, inferoseptal, inferior, inferolateral and anterolateral. Time-strain curves of individual segments are represented in the bottom panel. Peak circumferential strain value for each segment is calculated automatically.

\section{Statistical analysis}

Continuous variables were expressed as the mean \pm standard deviation. Nominal variables were expressed as percentages. Differences before and after chemotherapy were determined using paired sample t test for continuous variables and Fisher's exact test for categorical data. Multiple linear regression analyses to determine the predictors of LS, CS, RS were carried out, examined with age, hypertension, cigarette smoking, epirubicin dose during each session and cumulative dose. Because of a small group of diabetes, this cardiac co-morbidity was not included in the model. Inter- and intra-observer reproducibility of global LS, CS, RS, $\mathrm{T}_{\text {LS-SD }}, \mathrm{T}_{\mathrm{CS}-\mathrm{SD}}$ and $\mathrm{T}_{\mathrm{RS}-\mathrm{SD}}$ were assessed using intraclass correlation coefficients (ICCs). Data were analyzed by SPSS version 16.0 (SPSS, Inc, Chicago, IL, USA). A value of $\mathrm{p} \leq 0.05$ was considered significant.

\section{Results}

Four patients were excluded from the analysis because of poor image (defined as $>2$ non-visualized segments). Two died before completion of chemotherapy due to pneumonia and secondary respiratory failure. A total of 67 patients, 36 males, ranging in age from 18 to 76 years (mean age $52.58 \pm 13.86$ years) were finally included in the statistical analysis. Eleven patients had a history of hypertension and 3 diabetes, which were well-controlled by medication. Nineteen patients had a history of smoking or were current smokers. Cumulative dose of epirubicin was $254.15 \pm$ $\pm 95.29 \mathrm{mg} / \mathrm{m}^{2}$ (ranging from 149.03 to $561.69 \mathrm{mg} / \mathrm{m}^{2}$ ). After treatment of R-CHOP, no patient complained of cardiovascular related symptoms. EKG remained normal in all patients. In 1139 segments from 67 patients analyzed by means 
Table 1. Conventional echocardiographic parameters before and after chemotherapy.

\begin{tabular}{lccc}
\hline & Before & After & P \\
\hline Left ventricular ejection fraction [\%] & $63.56 \pm 2.38$ & $63.78 \pm 3.02$ & 0.67 \\
Fraction shortening [\%] & $34.30 \pm 2.76$ & $34.61 \pm 2.61$ & 0.45 \\
E velocity [m/s] & $64.00 \pm 13.78$ & $63.24 \pm 15.04$ & 0.82 \\
A velocity [m/s] & $70.80 \pm 15.39$ & $66.78 \pm 15.09$ & 0.09 \\
E/A ratio & $0.95 \pm 0.33$ & $0.99 \pm 0.32$ & 0.33 \\
Deceleration time [ms] & $191.78 \pm 44.37$ & $183.09 \pm 40.07$ & 0.30 \\
Isovolumic relaxation time [ms] & $87.69 \pm 15.94$ & $88.30 \pm 13.58$ & 0.34 \\
\hline
\end{tabular}

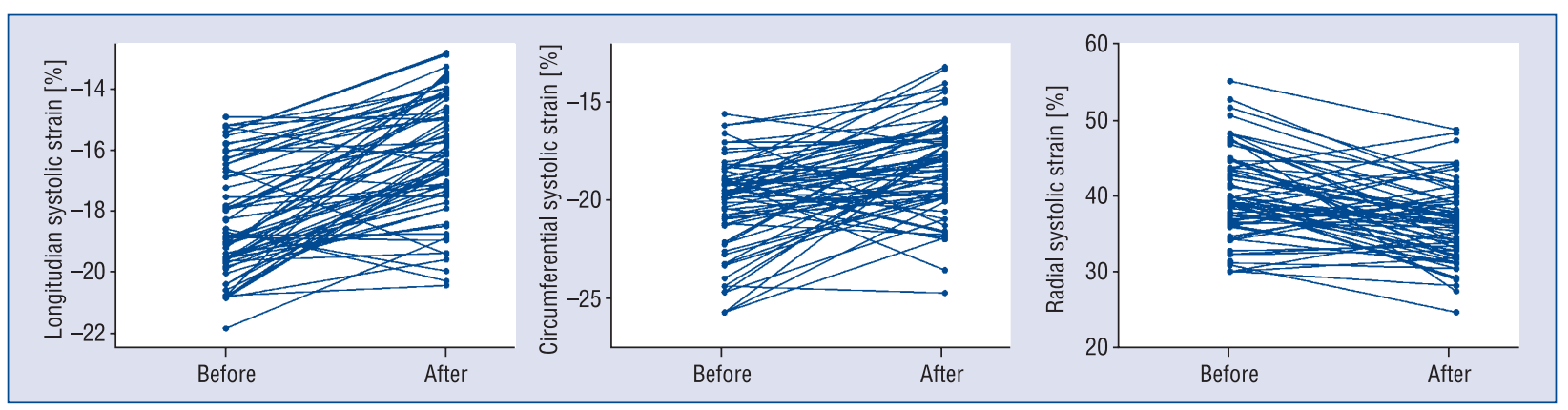

Figure 2. Peak systolic strain values before and after chemotherapy of individual patient.

of LS, $48(4.21 \%)$ segments were considered uninterpretable, and were primarily located in the anterior wall and apex $(87.50 \%$ of all uninterpretable segments). In 804 segments for CS and RS analysis, 16 non-trackable segments $(1.99 \%)$ were excluded, primarily located in the lateral wall (93.75\% of all non-trackable segments).

Conventional echocardiographic parameters

Table 1 summarizes the echocardiographic findings in patients before and after chemotherapy. Conventional parameters of systolic and diastolic function, including LVEF, fraction shortening, E velocity, A velocity, E/A ratio, deceleration time and isovolumic relaxation time showed no significant difference after epirubicin treatment.

\section{Two-dimensional speckle tracking echocardiography}

The peak longitudinal systolic strains of base, mid- and apical segments were significantly reduced after chemotherapy from $-16.57 \pm 2.42 \%$ to $-15.34 \pm 2.62 \%$ ( $\mathrm{p}<0.01),-19.34 \pm 3.23 \%$ to $-17.62 \pm 4.28 \%(\mathrm{p}<0.01),-18.72 \pm 3.54 \%$ to $-17.33 \pm 3.15 \%(\mathrm{p}=0.01)$, respectively. The peak circumferential systolic strains of base, mid-seg- ments $(-19.88 \pm 2.96 \%$ vs. $-18.64 \pm 2.91 \%,-20.50 \pm$ $\pm 3.74 \%$ vs. $-18.33 \pm 2.91 \%$, p all $<0.01$ ) and peak radial systolic strain of base and mid-levels (38.72 \pm $\pm 5.96 \%$ vs. $35.38 \pm 6.58 \%, 40.71 \pm 7.45 \%$ vs. $37.28 \pm 7.16 \%$, p all < 0.01 ) were markedly lower than those values before treatment.

With regard to the deformation of all the regional segments, global LS $(-18.30 \pm 1.87 \%$ vs. $-16.18 \pm 1.92 \%, \mathrm{p}<0.01)$, CS $(-20.37 \pm$ $\pm 2.89 \%$ vs. $-18.25 \pm 2.40 \%, \mathrm{p}<0.01), \operatorname{RS}(39.95 \pm$ $\pm 5.79 \%$ vs. $36.15 \pm 5.79 \%, p<0.01$ ) were statistically different compared with the baseline values (Figs. 2, 3).

Taking into account the dyssynchrony parameters of $\mathrm{LV}, \mathrm{T}_{\mathrm{LS}-\mathrm{SD}}, \mathrm{T}_{\mathrm{CS}-\mathrm{SD}}$ and $\mathrm{T}_{\mathrm{RS}-\mathrm{SD}}$ showed no significantly difference before and after chemotherapy (Table 2).

\section{Determinants of peak myocardial strain}

On multivariate analysis, among age, hypertension, cigarette smoking, epirubicin dose during each session and cumulative dose, hypertension was the only independent predictor of reduction of global LS (beta $0.27,95 \%$ CI 2.68-0.16, $p=0.03$ ), global CS (beta $0.26,95 \%$ CI $4.25-0.14, \mathrm{p}=0.04$ ) and global RS (beta $0.26,95 \%$ CI 8.94-0.34, $\mathrm{p}=0.04$ ). 


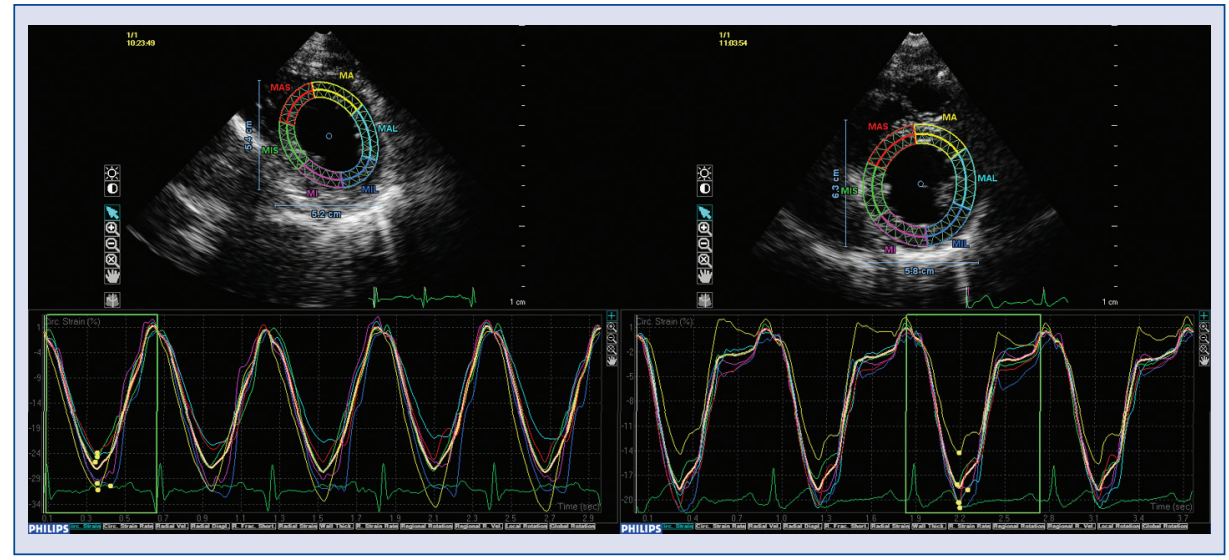

Figure 3. Circumferential strain curves of mid-papillary level before (left panel) and after (right panel) chemotherapy in the same patient. Peak circumferential strain reduced from $-27.57 \%$ to $-18.50 \%$ after epirubicin administration. However, time to peak strain showed no significant difference.

Table 2. Strain values and standard deviation of time to peak strains before and after chemotherapy administration.

\begin{tabular}{lccc}
\hline & Before & After & P \\
\hline Global LS [\%] & $-18.30 \pm 1.87$ & $-16.18 \pm 1.92$ & $<0.01$ \\
Global CS [\%] & $-20.37 \pm 2.89$ & $-18.25 \pm 2.40$ & $<0.01$ \\
Global RS (\%) & $39.95 \pm 5.79$ & $36.15 \pm 5.79$ & $<0.01$ \\
$T_{\text {LS-SD }}$ & $4.96 \pm 0.95$ & $5.38 \pm 1.86$ & 0.47 \\
$T_{\text {CS-SD }}$ & $4.64 \pm 1.26$ & $4.94 \pm 1.65$ & 0.65 \\
$T_{\text {RS-SD }}$ & $5.27 \pm 1.86$ & $5.08 \pm 1.36$ & 0.92 \\
\hline
\end{tabular}

CS — circumferential strain; LS - longitudinal strain; RS — radial strain; $T_{\text {CS-SD }}$ - standard deviation of time to peak circumferential strains of 12 segments; $T_{\text {LS-SD }}$ - standard deviation of time to peak longitudinal strains of 17 segments; $T_{\text {RS-SD }}-$ standard deviation of time to peak radial strains of 12 segments

\section{Inter- and intra-observer variation}

Inter-observer measurement showed ICC $=$ $=0.85$ for $\mathrm{LS}, 0.82$ for $\mathrm{T}_{\mathrm{LS}-\mathrm{SD}}, 0.92$ for $\mathrm{CS}, 0.96$ for $\mathrm{T}_{\text {CS-SD }}, 0.89$ for RS, 0.87 for $\mathrm{T}_{\mathrm{RS}-\mathrm{SD}}$. Similarly, intra-observer measurement showed ICC $=0.89$ for $\mathrm{LS}, 0.86$ for $\mathrm{T}_{\text {LS-SD }}, 0.98$ for CS, 0.96 for $\mathrm{T}_{\text {CS-SD }}$, 0.94 for RS, 0.92 for $\mathrm{T}_{\mathrm{RS}-\mathrm{SD}}$, indicating satisfactory reproductivity by 2D speckle tracking (Fig. 4).

\section{Discussion}

Anthracyclines, such as doxorubicin and epirubicin, have been used extensively and continue to be some of the most active cytotoxic agents available to treat a wide spectrum of hematologic malignancies and solid tumors, including acute myeloid, lymphoid leukemia, lymphoma and breast cancer. As a result of advances in the treatment of cancers, the number of survivors will fortunately continue to increase.
Unfortunately, despite their success in treating cancer, anthracyclines are limited by their serious toxic effects on the myocardium. Life altering cardiac sequelae from anthracyclines remain a problem, with a range of $5-23 \%$ of patients developing late-onset heart failure secondary to anthracycline-induced cardiotoxicity [10]. Reliable, sensitive and non-invasive method in detecting cardiac function is of vital importance during anthracycline administration.

Strain is a dimensionless parameter representing deformation of a myocardial segment relative to its original dimensions within a systolic time-frame. With standard 2D echocardiographic gray-scale imaging, reflected ultrasound gives rise to speckles of bright signal within the myocardium. Frame-by-frame tracking of the movement of these natural acoustic markers throughout the cardiac cycle allows assessment of temporal displacement between neighboring markers and quantification 


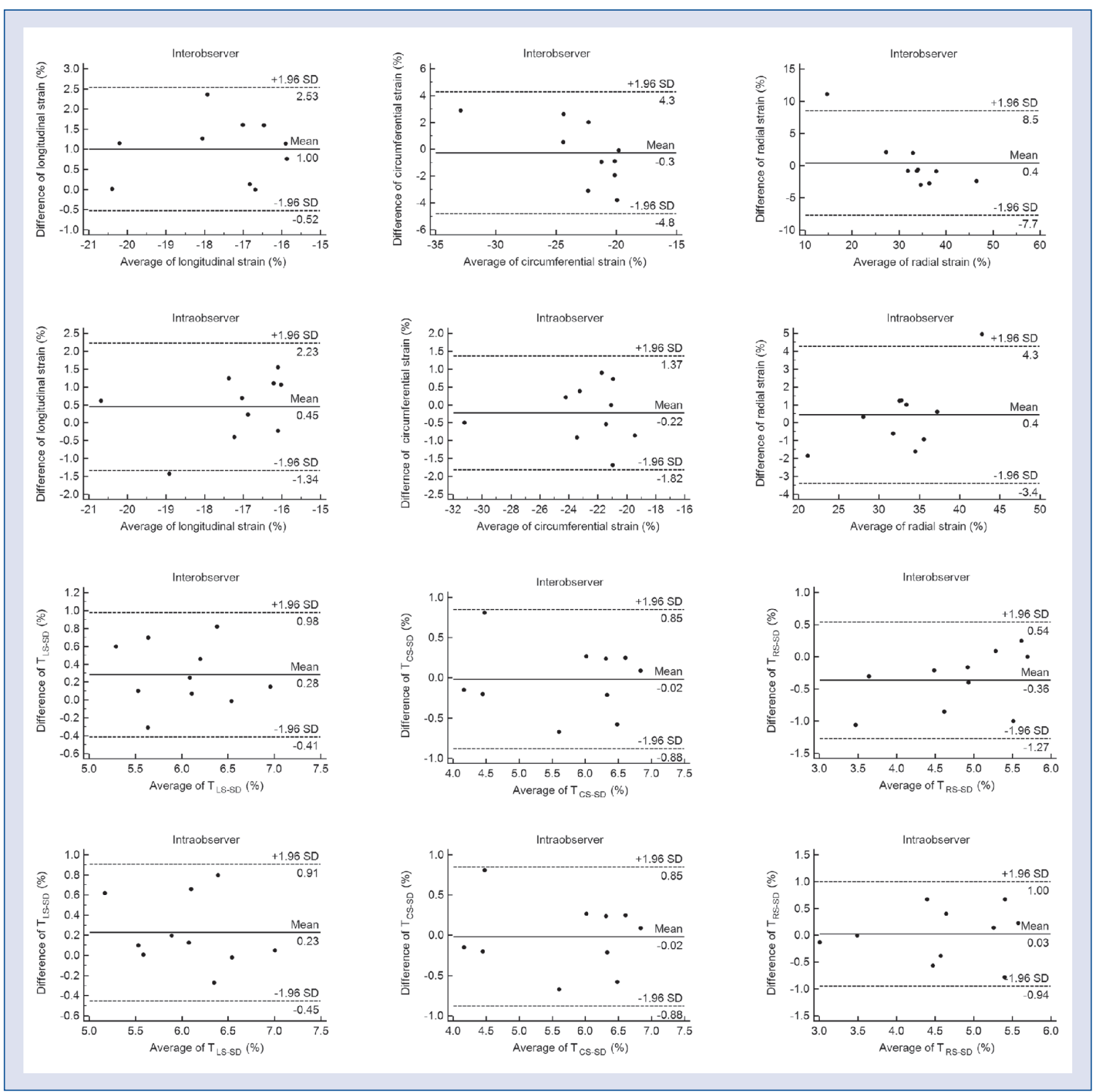

Figure 4. Bland-Altman analysis for intra-observer and inter-observer reliability.

of myocardial deformation. With the advantages of angle independence, speckle tracking echocardiography, allowing comprehensive assessment of both regional and global LV myocardial deformation, may provide reliable and sensitive parameter for early cardiotoxicity detection.

In a cross-sectional study, Ho et al. [11] found that compared with normal volunteers, subclinical systolic myocardial abnormalities were present in asymptomatic breast cancer survivors up to 6 years after anthracycline treatment using speckle tracking echocardiography, which was proved by other speckle tracking echocardiography-derived study [12]. However, there was a limited study demonstrating early subclinical cardiotoxicity in patients receiving anthracycline. Importantly, this non-cross-sectional study showed evidence of reduced 2D speckle tracking echocardiography-derived global and regional systolic function in asymptomatic individuals shortly after epirubicin administration. Cardiotoxicity that occurs soon after anthracycline administration may be a harbinger of later toxicity. Thus, early detection of cardiotoxicity will enable to balance between the 
cardiac risk and the potential cancer treatment benefits in an individual patient. Our study may present a paradigm shift in the monitoring of anthracycline cardiotoxicity from conventional assessment of changes in the LV cavity dimension to direct assessment of myocardial deformation. A recent study showed that even children who have received a cumulative doxorubicin dose as low as $45 \mathrm{mg} / \mathrm{m}^{2}$ had reduced LV mass, implying the absence of a safe dose that was free of cardiotoxicity [1]. We also found that though the mean cumulative dose of epirubicin in our study was only $254.15 \pm$ $\pm 94.15 \mathrm{mg} / \mathrm{m}^{2}$ (ranging from 561.69 to $149.03 \mathrm{mg} / \mathrm{m}^{2}$ ), quite lower than the recommended maximum lift time cumulative dose [13], subclinical cardiac systolic dysfunction was still observed in those patients, in accordance with the demonstration that anthracycline damage to all cardiac structures may begin with the first anthracycline dose [14]. Stanton et al. [15] concluded that in patients with LV dysfunction, global LS measured by 2D strain was superior to ejection fraction and wall motion score index for the prediction of long-term outcome in a large, clinical population. Thus, we believed that these strain parameters in early echocardiographic surveillance during the administration of anthracycline might facilitate the early detection of myocardial dysfunction for timely intervention.

In our study, the RS, as well as LS and CS showed significant reduction after chemotherapy, which was in concordance with some previous studies $[12,16]$, however, discordant with others $[11,17]$. Ho et al. [11] demonstrated that there was no significant difference in RS between the chemotherapy and control groups. There are many reasons accounting for the discordance. Some of those studies used tissue Doppler imaging-derived parameters of deformation, where global radial cardiac performance was only assessed by fractional shortening [17]. Secondly, it was recently published that peak RS differed largely between different software and algorithms, small changes in width can change large RS differences [18]. Hence, it was believed that different modalities, machines and algorithms may account for the discordant results.

In spite of a considerable interest in identifying risk factors for subclinical cardiotoxicity, findings have often been conflicting. Hypertension, which was associated with LV remodeling and dysfunction, was an independent predictor of myocardial strain in our group. The exact mechanisms of anthracycline-induced cardiotoxicity have not yet been fully elucidated, however, oxidative stress may have a central role [13]. It has been estab- lished that hypertension is associated with high levels of oxidative stress [19], which may potentially augment anthracycline-induced myocardial dysfunction. It emphasizes the importance of life-long control of cardiac risk factors especially in those long-term survivors. Established methods to prevent and modulate anthracycline cardiotoxicity include adding angiotensin-converting enzyme inhibitors prior to anthracycline exposure in patients with hypertension [20].

Cheung et al. [12] proved that dyssynchronous $\mathrm{LV}$ contraction was documented using real-time 3D echocardiography in children after anthracycline therapy. However, in our study, we found no significant difference shortly after epirubicin administration evaluated by $\mathrm{T}_{\text {LS-SD }}, \mathrm{T}_{\text {CS-SD }}$ and $\mathrm{T}_{\text {RS-SD }}$ in adult patients. Speckle tracking echocardiography and real-time 3D echocardiography provide distinctly different measures of ventricular timing and cannot be considered directly comparable for assessing intraventricular dyssynchrony [21]. Furthermore, the cardiac damage induced by anthracycline progresses with time and young children are more susceptible to the injury. In our study we demonstrated the early effect of epirubicin on adults, which may explain the contradistinction between these 2 studies. Long-term large-scale study is required to evaluate the effect of anthracycline on LV mechanical dyssynchrony.

\section{Limitations of the study}

Several limitations to this study warrant comment. The present study does not provide information on the value of myocardial deformation parameters in prognostication. Further out-come studies with hard clinical end points will be required to determine the clinical significance of our findings. Secondly, although speckle tracking echocardiography allows interrogation of global strain, these parameters are not entirely load-dependent and need to be interpreted with caution when an alteration of cardiac status with acute changes in load is anticipated.

\section{Conclusions}

Despite normal LVEF, subtle abnormalities in myocardial systolic function were present in patients shortly after anthracycline exposure. Speckle tracking echocardiography, a potential non-invasive tool for the early detection of subclinical anthracycline-induced myocardial abnormalities, might facilitate the early intervention of myocardial dysfunction in these patients. 


\section{Acknowledgements}

This work was supported by grants No. 81201095, No. 30972812 and No. 81000614 from the National Nature Science Foundation of China.

\section{Conflict of interest: none declared}

\section{Reference}

1. Lipshultz SE, Lipsitz SR, Sallan SE et al. Chronic progressive cardiac dysfunction years after doxorubicin therapy for childhood acute lymphoblastic leukemia. J Clin Oncol, 2005; 23: 2629-2636.

2. van Dalen EC, van den Brug M, Caron HN, Kremer LC. Anthracycline-induced cardiotoxicity: comparison of recommendations for monitoring cardiac function during therapy in paediatric oncology trials. Eur J Cancer, 2006;42: 3199-3205.

3. Villani F, Meazza R, Materazzo C. Non-invasive monitoring of cardiac hemodynamic parameters in doxorubicin-treated patients: Comparison with echocardiography. Anticancer Res, 2006; 26: 797-801.

4. Walker J, Bhullar N, Fallah-Rad N et al. Role of three-dimensional echocardiography in breast cancer: comparison with two-dimensional echocardiography, multiple-gated acquisition scans, and cardiac magnetic resonance imaging. J Clin Oncol, 2010; 28: 3429-3436.

5. Amundsen BH, Helle-Valle T, Edvardsen T et al. Noninvasive myocardial strain measurement by speckle tracking echocardiography: Validation against sonomicrometry and tagged magnetic resonance imaging. J Am Coll Cardiol, 2006; 47: 789-793.

6. Cho GY, Chan J, Leano R, Strudwick M, Marwick TH. Comparison of two-dimensional speckle and tissue velocity based strain and validation with harmonic phase magnetic resonance imaging. Am J Cardiol, 2006; 97: 1661-1666.

7. Helle-Valle T, Crosby J, Edvardsen T et al. New noninvasive method for assessment of left ventricular rotation: Speckle tracking echocardiography. Circulation, 2005; 112: 3149-3156.

8. Reisner SA, Lysyansky P, Agmon Y, Mutlak D, Lessick J, Friedman Z. Global longitudinal strain: A novel index of left ventricular systolic function. J Am Soc Echocardiogr, 2004; 17: 630-633.

9. Lang RM, Bierig M, Devereux RB et al.; American Society of Echocardiography's Nomenclature and Standards Committee; Task Force on Chamber Quantification; American College of Cardiology Echocardiography Committee; American Heart Association; European Association of Echocardiography, European
Society of Cardiology.Recommendations for chamber quantification. Eur J Echocardiogr, 2006; 7: 79-108.

10. Cardinale D, Colombo A, Lamantia G et al. Anthracycline-induced cardiomyopathy: Clinical relevance and response to pharmacologic therapy. J Am Coll Cardiol, 2010; 55: 213-220.

11. Ho E, Brown A, Barrett P et al. Subclinical anthracycline- and trastuzumab-induced cardiotoxicity in the long-term follow-up of asymptomatic breast cancer survivors: A speckle tracking echocardiographic study. Heart, 2010; 96: 701-707.

12. Cheung YF, Hong WJ, Chan GC, Wong SJ, Ha SY. Left ventricular myocardial deformation and mechanical dyssynchrony in children with normal ventricular shortening fraction after anthracycline therapy. Heart, 2010; 96: 1137-1141.

13. Barry E, Alvarez JA, Scully RE, Miller TL, Lipshultz SE. Anthracycline-induced cardiotoxicity: Course, pathophysiology, prevention and management. Expert Opin Pharmacother, 2007; 8: 1039-1058.

14. Lipshultz SE, Rifai N, Sallan SE et al. Predictive value of cardiac troponin $\mathrm{T}$ in pediatric patients at risk for myocardial injure. Circulation, 1997; 96: 2641-2648.

15. Stanton T, Leano R, Marwick TH. Prediction of all-cause mortality from global longitudinal speckle strain: Comparison with ejection fraction and wall motion scoring. Circ Cardiovasc Imaging, 2009; 2: 356-364.

16. Tassan-Mangina S, Codorean D, Metivier M et al. Tissue Doppler imaging and conventional echocardiography after anthracycline treatment in adults: Early and late alterations of left ventricular function during a prospective study. Eur J Echocardiogr, 2006; 7: 141-146.

17. Ganame J, Claus P, Uyttebroeck A et al. Myocardial dysfunction late after low-dose anthracycline treatment in asymptomatic pediatric patients. J Am Soc Echocardiogr, 2007; 20: 1351-1358.

18. Biaggi P, Carasso S, Garceau P et al. Comparison of two different speckle tracking software systems: Does the method matter? Echocardiography, 2011; 28: 539-547.

19. Gönenç A, Hacışevki A, Tavil Y, Cengel A, Torun M. Oxidative stress in patients with essential hypertension: A comparison of dippers and non-dippers. Eur J Intern Med, 2013; 24: 139-144.

20. Geisberg CA, Sawyer DB. Mechanisms of anthracycline cardiotoxicity and strategies to decrease cardiac damage. Curr Hypertens Rep, 2010; 12: 404-410.

21. Faletra FF, Conca C, Klersy C et al. Comparison of eight echocardiographic methods for determining the prevalence of mechanical dyssynchrony and site of latest mechanical contraction in patients scheduled for cardiac resynchronization therapy. Am J Cardiol, 2009; 103: 1746-1752. 